\title{
Effects of frequency parameter due to elastic wave in monoclinic elastic medium
}

\author{
J. Lalvohbika* \\ Department of Mathematics, Pachhunga University College, Aizawl 796001, Mizoram, INDIA \\ Corresponding author: vohbikajongte@pucollege.edu.in
}

\begin{abstract}
The propagation of elastic waves has many applications especially in geophysics and engineering. They are used in the detection of notches and faults in different types of materials such as in railway tracks, buried landmines, seismic activities, and exploration of valuable materials such as minerals, crystals, natural gas, etc. When the seismic signal passes through different discontinuities inside the earth, the phenomena of reflection and transmission takes place. In this paper, the phenomenon of reflection and transmission of elastic waves due to incident qSV-wave at a corrugated interface between two different monoclinic elastic half-spaces are discussed. There exist an irregularly reflected and transmitted waves due to undulated nature of the interface, besides the regularly reflected and transmitted ones. Using Rayleigh's method of approximation, the expressions for the reflection and transmission coefficients of the regular and irregular waves are obtained. It is observed that these coefficients are functions of angle of incidence and elastic constants and they are greatly influenced by the frequency and corrugation parameter. For a special type of interface, $\mathrm{x}_{3}=\mathrm{d} \cos \mathrm{px}_{2}$ we have computed these coefficients and discussed the effects of frequency parameter.
\end{abstract}

Keywords: qSV and qP-waves, monoclinic medium, reflection and transmission coefficients, corrugated interface, phase velocity.

\section{INTRODUCTION}

Elastic wave propagation is a very interesting topic that deals with numerous problems in various fields such as earthquake engineering, geophysics, signal processing, echography and metallurgy. They are useful in detection of notches and faults in different types of materials such as in railway tracks, buried land-mines, etc. The technique of seismic wave propagation is a tool for investigating the internal structure of the earth and also used for exploration of valuable materials such as minerals, crystals, fluids (oils, water) etc. beneath the earth surface. When the seismic signal passes through different discontinuities inside the earth, the phenomena of reflection and transmission takes place. These signals carry lots of information about the earth's structures and many such problems are in open literatures, i.e. Chattopadhyay and Choudhury (1995), Chattopadhyay et al. (1997), Dowaikh and Ogden (1991), Sheriff and Geldart
(1995), Singh (1999), Singh and Khurana (2002), Udias (1999) and others.

Singh and Khurana (2001) investigated the reflection and transmission of $P$ and SV-waves at the interface between two monoclinic elastic half-spaces. Singh and Tomar (2007) investigated the problem of qP-waves at a corrugated interface between two dissimilar monoclinic elastic half-spaces and obtained the reflection and transmission coefficients of the irregular waves using Rayleigh's technique. Kaur and Tomar (2004) investigated the problem of reflection and transmission of shear wave incident upon a corrugated interface between two monoclinic elastic half-spaces with the help of Rayleigh's technique. But, there are many experimental evidences that the interfaces between layers are not perfectly plane, but they are of irregular in nature. These irregular natures of the interfaces affect in the reflection and transmission of elastic waves. Thus, it is important to take into account the problems related with the effects 
of irregular interfaces.

It was Rayleigh (1907) who first discussed the problems on reflection and transmission phenomena of light/ sound waves from an irregular boundary surface. In his method, the function defining the corrugated interface in the expressions of boundary conditions is expanded in Fourier series and the unknown coefficients are determined up to any given order of approximation in terms of small parameter characteristics of magnitude and slope of corrugated interface. Later, Rayleigh's technique was applied to various other fields to study the reflection and transmission phenomena of waves at irregular boundary surface. Several papers on scattering of elastic waves from boundary surfaces have been appeared based on Rayleigh's method and other techniques such as Abubakar (1962), Asano (1966), Dunkin and Eringen (1991), Gupta (1978), Kuo and Nafe (1962), Levy and Deresiewicz (1967), Rice (1951), Singh and Tomar $(2007,2008)$ and Tomar and Kaur $(2003)$.

\section{BASIC EQUATIONS}

The constitutive relation in a homogeneous anisotropic elastic material of monoclinic type with 23 -plane as the plane of symmetry are given by Singh and Khurana (2001)

$$
\begin{aligned}
& 11=1111+1222+1333+2_{1423,}, \quad 22= \\
& 1211+2222+2333+22423 \text {, } \\
& 23=1411+2422+3433+24423 \text {, } \\
& 33=1311+2322+3333+23423,12= \\
& 25513+5612 \text {, } \\
& 2\left(\begin{array}{lll}
56 & 13 & + \\
66 & 12
\end{array}\right)
\end{aligned}
$$

are stress tensors, $\quad(,=1,2,3, \ldots, 6)$ are elastic constants and is the strain tensor given by

$$
=\frac{1}{2}-+- \text {. }
$$

The equation of motion in such anisotropic materials without body forces are given by

$$
-=\frac{2}{2}, \quad(,=1,2,3)
$$

where is density of the medium.

The equations of motion in terms of displacements components may be written as

$$
\begin{aligned}
& 22 \frac{2}{2^{2}}+44 \frac{2}{3^{2}}+24 \frac{2}{2^{2}}+34 \frac{2}{3^{2}}+ \\
& 224 \frac{2 \frac{2}{23}}{22^{3}}+23+24 \frac{23}{23}=\frac{22}{2} \text {, }
\end{aligned}
$$

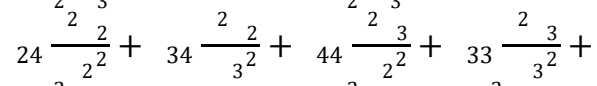

$$
\begin{aligned}
& 234 \frac{23}{23}+23+24 \frac{2 \frac{2}{23}}{23}=\frac{23}{2},
\end{aligned}
$$

The solution of these equations may be taken in the form

$$
2,32,3,=
$$

where $\mathrm{c}$ is the phase velocity, $\mathrm{k}$ the wavenumber, $=(0$, $2,3)$ is the unit propagation vector, $=\left(\begin{array}{lll}0, & 2,3\end{array}\right)$ is the unit displacement vector.

Using these expressions of 2 and 3 into Eqs. (2.3) and (2.4), we have

$$
\left.{ }_{3}^{2}\right)_{3}=0, \quad 2 \quad 2+\quad 3=0, \quad 2+(
$$

where

$$
\begin{aligned}
& =22 \stackrel{2}{2}+44 \stackrel{2}{3}+22423 \text {, = } \\
& 24 \stackrel{2}{2}+34 \stackrel{2}{3}+23+4423 \text {, } \\
& =44 \stackrel{2}{2}+33_{3}^{2}+23423 \text {. }
\end{aligned}
$$

Using Eq. (2.6), we get the phase velocity as

$$
22_{2,1}^{2}=+ \pm \overline{(-)^{2}+4^{2}}
$$

where + sign is for $\quad$-wave and - sign is for -wave.

\section{Problem formulation}

Consider the Cartesian coordinates with 1 and 2 -axis lying horizontal and 3 -axis as vertical with positive direction pointing downward. Suppose two dissimilar homogeneous monoclinic half-spaces, = $\{2 ; 3: 2 \in, 3 \in \zeta, \infty\}$ and ${ }^{\prime}=\{(2 ; 3):$ $\left.{ }_{2} \in, 3 \in(-\infty, \zeta)\right\}$ are separated by $3=(2)$, which is a periodic function of $y$ independent of $x$ whose mean value is zero. We will denote all elastic constants, stress tensors and displacement components in medium, $\mathrm{M}$ without prime and those of $\mathrm{M}^{\prime}$ with primes. The geometry of the problem is shown by Figure 1 . The Fourier series expansion of $(2)$, is given as

$$
2={ }_{=1}^{\infty} \quad \text { in } 2+
$$

where and _ are the coefficients of series expansion of order $n, p$ is the wavenumber and $=\overline{-1}$.

Introduce constants ; ; and as

so that

$$
\pm 1=\frac{7}{2}, \pm=\frac{\mp}{2}, \quad(=2,3,4, \ldots \ldots)
$$

$$
\stackrel{\infty}{\infty} \quad \cos 2+\sin ^{2} 2^{2} .
$$

If the interface is represented by only one cosine term,

i.e. $(2)=\cos \left(p_{2}\right)$, then the wavelength of corrugation is 2 / and $d$ is the amplitude of corrugation.

We shall now discuss the reflection and transmission of elastic waves due to incident plane qSV-wave at the corrugated interface, ${ }_{3}=(2)$. Suppose a plane qSVwave propagating in the half-space, $M$ with an angle 0 and amplitude constant ${ }_{0}$ be incident at the corrugated interface. This incident wave give rises regularly and irregularly reflected and transmitted $\mathrm{qSV}$ and $\mathrm{qP}$-waves [2]. 
The full structures of reflected and transmitted waves are given by:

(for the half-space, $M$ )

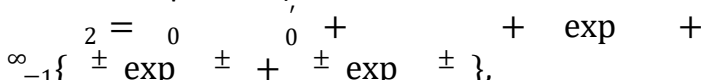

$$
\begin{aligned}
& \infty_{=1}\{ \pm \exp \pm+ \pm \exp \pm\} \text {, }
\end{aligned}
$$

where $(A, D)$ are amplitude constants of the regularly reflected qSV-wave at angle ,, \pm \pm are amplitude constants of the irregularly reflected qSV-waves at angles

,$\pm(B, E)$ are amplitude constants of the regularly reflected $\mathrm{qP}$-wave at angle,, \pm \pm are amplitude constants of the irregularly reflected $\mathrm{qP}$-waves at angles

\pm and the expressions of $0, \quad, \pm, Q, \pm$ are given by

$$
\begin{aligned}
& { }_{0}^{\prime}=\iota \omega-\frac{20-3 c o \quad 0}{0},=\iota \omega- \\
& \frac{2 \sin +3 \cos }{2}, \pm=\iota \omega-\frac{2 \pm_{+3} c o \pm}{2} \text {, } \\
& =\iota \omega-\frac{2 \sin +3 \cos }{1} \text { and } \pm=\iota \omega \quad- \\
& \frac{2 \sin \pm_{+3} \text { co } \quad \pm}{1} \text {. }
\end{aligned}
$$

These amplitude constants satisfy the following relations [21]

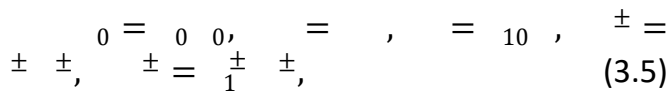

where

$$
\begin{gathered}
0=\frac{0}{2-0}, \quad=\frac{10}{2-10}, \quad 10=\frac{20}{2-20}, \quad \pm= \\
\frac{1 \pm}{\frac{1}{2}-\frac{ \pm}{1}}, \quad \frac{ \pm}{1}=\frac{\frac{2}{2}}{\frac{2}{1}-\frac{ \pm}{2}},
\end{gathered}
$$

$22_{0}^{2}=0+0-\overline{0-0^{2}+40_{0}^{2}}, 22_{2}^{2}=10+$ $10-\frac{0}{10-10^{2}+4 \frac{10}{10}}$

$2 \stackrel{2}{2}=20+20+\overline{(20-20)^{2}+4 \underset{20}{2}}$.

(for the half-space, $\mathrm{M}^{\prime}$ )

$$
\begin{aligned}
& i_{2}^{1}=(\text { ) }+\quad()+ \\
& { }_{=1}^{\infty}\{ \pm \pm(0 \pm \pm\}
\end{aligned}
$$

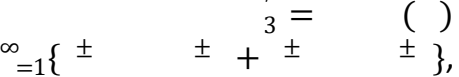

$$
\begin{aligned}
& ()+
\end{aligned}
$$

where $(G, I)$ are amplitude constants of the regularly transmitted qSV-wave at angle ,, \pm \pm are amplitude constants of the irregularly transmitted qSV-waves at angles,$\pm(H, J)$ are amplitude constants of the regularly transmitted qP-wave at angle ,, \pm \pm are amplitude constants of the irregularly transmitted $\mathrm{qP}$-waves at angles \pm and the expressions of,,$\pm \quad \pm$ are given

$$
\begin{aligned}
& \text { by }=\iota \omega-\frac{2 \sin -3 \cos }{2}, \pm=\iota \omega \\
& \frac{2 \pm_{-3} c o \pm}{1}, \quad=\iota \omega-\frac{2 \sin -3 \cos }{1} \\
& \pm=\iota \omega\left(-\frac{2}{ \pm_{-3} c o \pm} \frac{ \pm}{1}\right) .
\end{aligned}
$$

These amplitude constants also satisfy the following relations

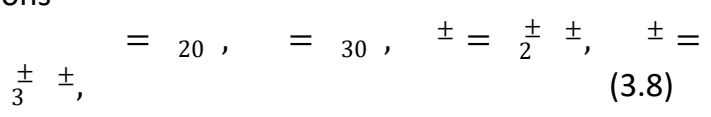

where

$$
\begin{aligned}
& 20=\frac{30}{\prime^{\prime 2}-30}, \quad 30=\frac{40}{\prime^{\prime 2}-40}, \quad \frac{ \pm}{1}= \\
& \frac{\frac{3}{3}}{, 2_{2}^{2}-\frac{ \pm}{3}}, \quad \frac{ \pm}{3}=\frac{\frac{ \pm}{4}}{1_{1}^{2}-\frac{ \pm}{4}} \\
& 2 \stackrel{2}{2}=30+30- \\
& \frac{30-30^{2}+4 \frac{3}{30}}{(40-40)^{2}+4_{40}^{2}}, \quad 2^{\prime}{ }_{1}^{\prime 2}={ }_{40}+{ }_{40}+
\end{aligned}
$$

The Snell's law which gives the relation of angle of incident, reflected as well as transmitted waves is given by Asano (1966)

$$
\frac{0}{0(0)}=\frac{\sin }{2()}=\frac{\sin }{1()}=\frac{\sin }{\frac{1}{2(}()}=\frac{\sin }{1(()}=\frac{1}{,}
$$

where is apparent velocity.

Moreover, Spectrum theorem gives the relations between the angle of regular wave and those of irregular waves Abubakar (1962)

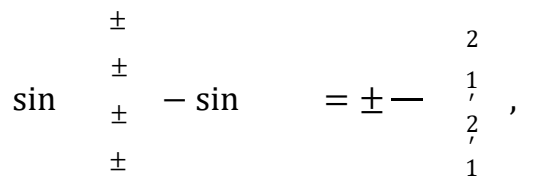

$$
\begin{aligned}
& =1,2, \ldots .
\end{aligned}
$$

\section{Boundary condition}

The component of displacements and tractions (normal and shear) are continuous at the corrugated interface. Mathematically, these conditions at $3=$ ( 2) may be written as

$$
\begin{aligned}
& { }_{2}={ }_{2}^{\prime}, \quad 3={ }_{3}^{\prime}, \\
& , 32+\left(3,{ }_{13}-22\right)^{\prime}-23^{\prime} 2={ }_{32}^{\prime}+\left(\begin{array}{c}
\prime \\
33
\end{array}-\right. \\
& \text { 22) }-23{ }^{\prime}, \quad \text { (4.2) } \\
& 33^{2} 2^{\prime}+22^{\prime 2}={ }_{33}^{\prime}-2_{23}^{\prime}{ }^{\prime}+{ }_{22}^{\prime} 2 \text {, }
\end{aligned}
$$

where 'is the derivative of with respect to . Inserting Eq. (2.1) into (4.2) and (4.3), we get

$\left\{(23-22)^{\prime}+{ }_{24}\left(1-{ }^{\prime} 2\right)\right\} \frac{2}{2}+\left\{(33-23)^{\prime}+\right.$ $\left.{ }_{34}\left(1-{ }^{\prime}\right)\right\} \frac{3}{3}+\left\{34-244^{\prime}+{ }_{44} 1-{ }^{\prime 2} \quad\left(\frac{2}{3}+\right.\right.$ $\left.\frac{3}{2}\right)=\left\{\left(\begin{array}{l}\prime 23 \\ 232\end{array}\right)^{\prime}+{ }_{24}^{\prime}\left(1-{ }^{\prime} 2\right)\right\} \frac{2}{2}+\left\{\left(_{33}^{\prime}-\right.\right.$

$$
\left.\left.{ }_{23}^{\prime}\right)^{\prime}+{ }_{34}^{\prime}\left(1-{ }^{\prime} 2\right)\right\} \frac{3}{3}+\left\{\left(\begin{array}{l}
\prime \\
34
\end{array}-{ }_{24}^{\prime}+{ }_{44}^{\prime}(1-\right.\right.
$$$$
\left.\left.{ }^{\prime} 2\right)\right\}\left(\frac{2}{3}+\frac{3}{2}\right) \text {, }
$$

$$
\begin{aligned}
& \left.\left\{23+22^{\prime 2}-2_{24}\right\}^{\prime}\right\} \frac{2}{2}+\left\{33+23^{\prime 2}\right. \\
& \left.-2_{34}{ }^{\prime}\right\} \frac{3}{3}+\left\{34+24^{\prime 2}\right. \\
& \left.-2_{44}{ }^{\prime}\right\}\left(\frac{2}{3}+\right.
\end{aligned}
$$

$\left.\frac{3}{2}\right)=\left\{\begin{array}{l}\prime \\ 23\end{array}+{ }_{22}^{\prime}{ }^{\prime} 2-2_{24}^{\prime}{ }^{\prime}\right\} \frac{2}{2}+\left\{\begin{array}{l}\prime \\ 33\end{array}+{ }_{23}^{\prime}{ }^{\prime} 2-\right.$ $\left.2_{34}^{\prime}\right\} \frac{3}{3}+\left\{\begin{array}{l}\prime \\ 34\end{array}+\right.$ 


$$
\left.2^{\prime} 4^{\prime}-2_{44}^{\prime}\right\}\left(\frac{2}{3}+\frac{3}{2}\right) .
$$

Using Eqs.(3.3), (3.4)), (3.6), (3.7), (3.9) and (3.10) into (4.1), (4.4) and (4.5), we get

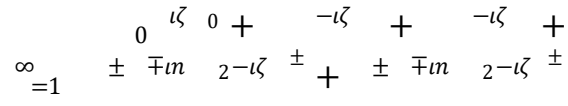

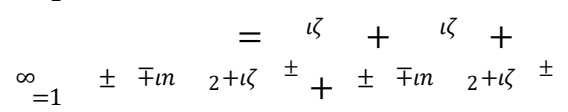

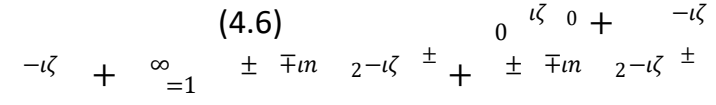

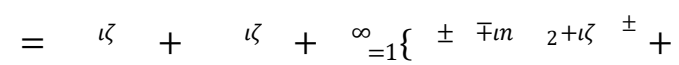$$
\left.\pm \mp i n \quad 2+\iota{ }^{ \pm}\right\}
$$

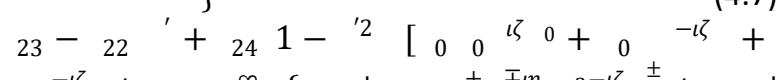$$
0^{-\iota \zeta+\quad} \infty_{=1}^{\infty}\{ \pm \quad \pm \mp m 2-\iota\}^{ \pm}+0 \pm
$$$$
\left.\left.\pm \mp \measuredangle n 2^{ \pm}\right\}\right]+33-23^{\prime}+341-{ }^{\prime} 2
$$

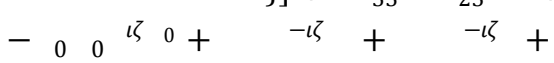$$
\infty_{=1}^{\infty} \pm \pm \mp i n \quad 2-\iota \zeta^{ \pm} \pm \pm \mp i n \quad 2-l \zeta^{ \pm}+
$$

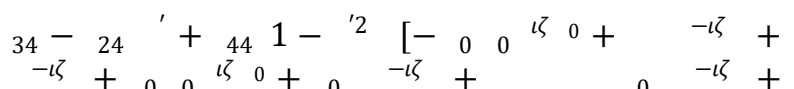

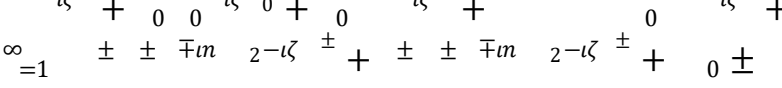$$
\left.\pm \mp i n \quad 2-\iota \zeta^{ \pm}+0 \pm \quad \pm \mp i n \quad 2-\iota \zeta^{ \pm}\right]=
$$

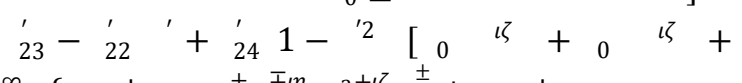$$
{ }_{=1}^{\infty}\{ \pm \quad \pm \mp i n 2+\iota\}^{ \pm}+0 \pm
$$$$
\left.\left.\pm \mp i n{ }^{2}+l \zeta^{ \pm}\right\}\right]+{ }_{33}-{ }_{23}^{\prime}+{ }_{34} 1-
$$$$
' 2 \zeta+\iota+\infty_{=1}^{\infty} \pm \pm \mp i n 2+\iota \zeta^{ \pm}
$$$$
\pm \pm \mp \curvearrowleft n 2+\iota \zeta^{\prime}{ }_{34}-{ }_{24}^{\prime}+{ }_{44}^{\prime} 1-{ }^{\prime} 2
$$$$
\zeta+\iota-0^{\zeta}-0^{\zeta}+
$$$$
{ }_{=1}^{\infty} \pm \pm-\imath 2^{-\imath \zeta^{ \pm}}+ \pm \pm \mp i n \quad 2+\iota \zeta^{ \pm}-0 \pm
$$$$
\pm \mp i n{ }_{2}+\zeta^{ \pm}-0 \pm \pm \mp i n 2+\iota \zeta^{ \pm}
$$

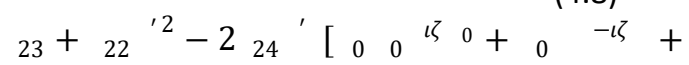

$$
\begin{aligned}
& 0-\iota \zeta+\infty_{=1}^{\infty}\left\{0 \pm \quad \pm \mp i n 2^{-\imath\}^{ \pm}}+0 \pm\right. \\
& \left.\left. \pm \mp i n \quad 2-\iota \zeta^{ \pm}\right\}\right]+33+23^{\prime 2}-
\end{aligned}
$$

$24^{\prime}-001 \zeta 0+-l \zeta+-l \zeta+$

$$
\left.{ }_{=1}^{\infty}\left\{ \pm \pm \mp n 2^{-l \zeta \pm}+ \pm \pm \mp m \quad 2-l \zeta^{ \pm}\right\}\right]+
$$

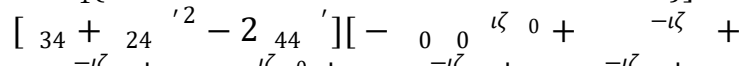$$
-\iota \zeta+00 \iota 0+0^{-l \zeta}+0^{-\iota \zeta+}
$$$$
\infty_{=1}^{\infty} \pm \pm \mp i n \quad 2-\iota \zeta^{ \pm}+ \pm \pm \mp i n \quad 2-\iota \zeta^{ \pm}+0 \pm
$$$$
\pm \mp i n 2^{-\imath \zeta}+0 \pm \pm \mp i n \quad 2-\iota \zeta^{ \pm}=
$$

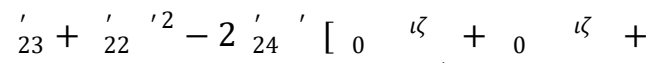

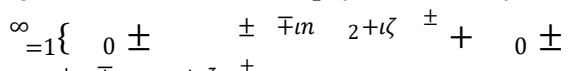

$\left.\left.\pm \mp i n \quad 2+\iota \zeta^{ \pm}\right\}\right]$

$2_{34}^{\prime}, \zeta+\zeta+\infty_{=1}^{\infty}\left\{ \pm \mp i n{ }_{2}+\iota \zeta^{ \pm}+\right.$

$$
\begin{aligned}
& 33+23^{\prime 2}-2_{34}^{\prime}, \quad \zeta+ \\
& \left.\infty_{=1}\left\{ \pm \pm \mp i n \quad 2+\iota \zeta^{ \pm}+ \pm \pm \mp i n \quad 2+\iota \zeta{ }^{ \pm}\right\}\right]-
\end{aligned}
$$

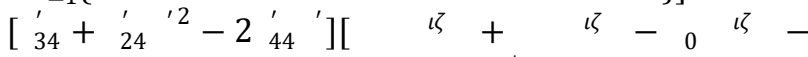

$$
\begin{aligned}
& 0 \iota+{ }_{=1}^{\infty} \pm \pm \mp i n \quad 2+\iota \zeta^{ \pm} \\
& \pm \pm \mp i n{ }^{2+l \zeta} \pm 0 \pm \quad \pm \mp i n{ }^{2}+\iota \zeta^{ \pm}-0 \pm \\
& \pm \mp i n n_{2}+\iota \zeta^{ \pm}
\end{aligned}
$$

where

$$
\begin{gathered}
0=\frac{\sin 0}{0}, \quad 0=\frac{\cos 0}{0}, \quad= \\
\frac{\cos }{2}, \pm=\frac{\cos \pm}{2, \quad=\frac{\cos }{1},} \\
\pm=\frac{\cos \pm}{1}, \quad=\frac{\cos }{2}, \pm=\frac{\operatorname{co} \pm}{2}, \\
=\frac{\cos }{1}, \quad \pm=\frac{\operatorname{co} \pm}{1}
\end{gathered}
$$

\section{Solution of first order approximation}

Assuming the amplitude of the corrugated interface is very small so that neglecting higher powers of , we have $\pm \iota 0=1 \pm \iota \zeta_{0}, \quad \pm \zeta=1 \pm \iota$, etc.

Using Eqs. (3.5), (3.8) and (5.1) into (4.6)-(4.9) and

collecting terms independent of and 2 , we obtain a set of equations

where

$$
\begin{aligned}
& \begin{array}{llll}
1 & 1 & -1 & -1
\end{array}
\end{aligned}
$$

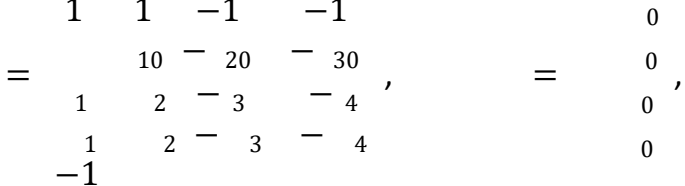

$$
\begin{aligned}
& \mathrm{T}=-0 \\
& -0 \\
& 0=024+440-\left(\begin{array}{lll}
0 & 44 & + \\
0 & & 0
\end{array}\right) \quad 0, \quad 1=24+ \\
& 440+(44+34) \text {, } \\
& { }_{2}=1024+440+\left(\begin{array}{lll}
10 & 44 & +34
\end{array}\right), \quad 3=2024+
\end{aligned}
$$

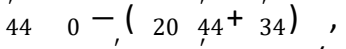

$$
\begin{aligned}
& 4=30{ }_{24}^{\prime}+{ }_{44}^{\prime} 0-\left(\begin{array}{ll}
30 & 4_{4}+{ }_{34}^{\prime}
\end{array}\right), \quad 0=0_{23}+ \\
& 340-\left(\begin{array}{ll}
0 & 34 \\
& + \\
33
\end{array}\right) \quad 0 \text {, } \\
& 1=23+340+(34+33), \\
& 2=1023+340+\left(\begin{array}{ll}
10 & 34 \\
10 & +33
\end{array}\right) \\
& 3=2023+, 34,0-\left(\begin{array}{ll}
20 & 34 \\
4_{3} & 1 \\
33
\end{array}\right), \quad 4=3023+ \\
& \begin{array}{ll}
34 & 0
\end{array}-\left(\begin{array}{ll}
30 & 34+ \\
+ &
\end{array}\right) \text {. }
\end{aligned}
$$

$$
-\frac{-}{0}=-\frac{-}{0}=-\frac{-}{0}=
$$

where the values of , and are obtained by replacing first, second, third and fourth column of with 
where the values of , and are obtained by replacing first, second, third and fourth column of with column matrix $T$ respectively. The ratios of the amplitude constants corresponding to horizontal components of displacement is obtained with the help of Eqs. (3.5), (3.8) and (5.3) as

$$
\frac{30}{0} \text { 一, }
$$

$$
-\frac{1}{0}=\frac{-}{0}-\frac{10}{0}-\frac{20}{0}=\frac{2}{0}, \frac{-}{0}=
$$

The reflection and transmission coefficients of reflected and transmitted $\mathrm{qSV}$ and $\mathrm{qP}$-waves for the incident $\mathrm{qSV}$ wave are given by

$$
\begin{aligned}
& =\frac{\overline{1+2}}{1+{ }_{0}^{2}}-\quad=\frac{\overline{1+2}}{1+2}- \\
& =\frac{\overline{1+2}}{1+20}-\quad=\frac{(5.5)}{1+\frac{2}{2}}-\frac{2}{1+{ }_{0}^{2}}-
\end{aligned}
$$

where $\overline{1+{ }_{0}^{2}} \quad 0$ is the amplitude of incident qSV-wave. We come to know that these coefficients depend on elastic constants and angle of incidence.

Next, comparing coefficients of ${ }^{\mp} \quad 2$ on the both the sides of those equations, we get

$$
\stackrel{\mp}{\mp}=\mp
$$

where

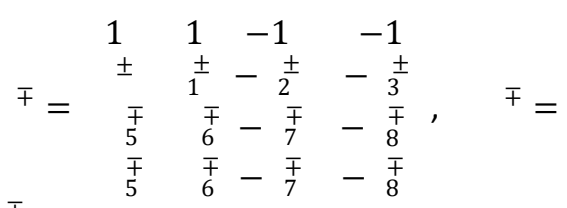$$
\begin{array}{ll} 
\pm & 0 \\
\pm & 0 \\
\pm & 0 \\
\pm & 0
\end{array} \quad \mp=\begin{gathered}
1^{\mp} \\
2^{\mp} \\
3^{\mp}
\end{gathered}
$$$$
1^{\mp}=\mp-0+\frac{-}{0}+\frac{-}{0}+\underset{0}{-}, 2^{\mp}=
$$$$
\mp \quad-00+\frac{0}{\mp}+10 \underset{0}{-}+20 \frac{0}{-}+30 \frac{0}{-}
$$$$
3^{\mp}=\underset{0}{\mp}+\underset{1}{\mp} \underset{0}{-}+\underset{2}{\mp} \underset{0}{\mp}-\underset{3}{\mp}-\underset{4}{\mp} \overline{0}_{0}, \quad 4^{\mp}=
$$$$
\stackrel{\mp}{\mp}+\underset{1}{\mp}-\underset{2}{\mp}-\underset{3}{\mp}-\underset{0}{\mp}-\underset{4}{\mp}, \quad \stackrel{\mp}{\mp}=
$$

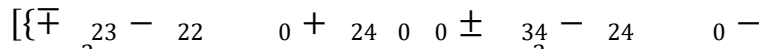

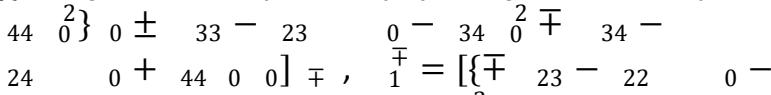$$
\left.240 \quad \mp \quad 34-24 \quad-4_{34}^{2}\right\} \quad \mp \quad 33-
$$

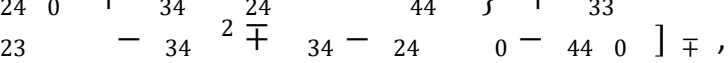$$
\stackrel{\mp}{\mp}=\mp_{23}-22 \quad 0-240 \quad \mp \quad 34-24
$$$$
{ }_{44}^{2}{ }_{10} \mp 33-23 \quad-34^{2} \mp{ }_{34}-
$$$$
{ }_{1}^{24} 00-440, \mp, \quad \stackrel{\mp}{=} \mp_{23}^{\prime}{ }_{2}^{\prime 2} 0+
$$$$
{ }_{1}^{24} 0 \pm 34-24,-, 4420 \pm 33-
$$

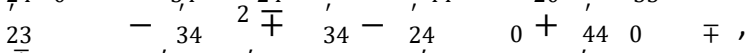

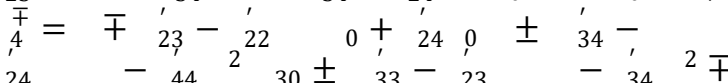

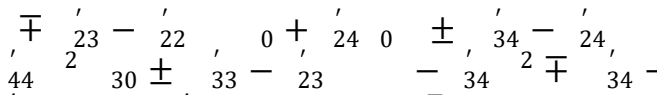

$$
\begin{aligned}
& \begin{array}{lllll}
44 & 30 \pm, 33-23 & -34 & + & 34 \\
24 & 0+440 & \mp, & 5=- & \pm 4
\end{array} \\
& 240 \pm \quad \pm+34^{ \pm}+440 \pm \quad, \quad \frac{\mp}{6}= \\
& -44^{ \pm}+240_{1} \quad 1^{ \pm}+34 \pm+440 \pm \\
& \mp={ }_{74}^{\mp} \pm-,{ }_{2}^{\prime} 40 \pm, \quad \frac{ \pm}{2}+{ }_{34}^{\prime} \pm-, 440 \pm \\
& \stackrel{\mp}{\mp}={ }_{44}^{\prime} \pm-240 \pm \frac{ \pm}{3}+3^{\prime} \pm-
\end{aligned}
$$

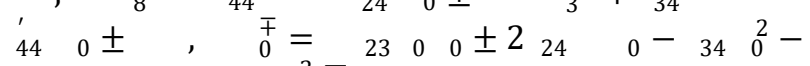

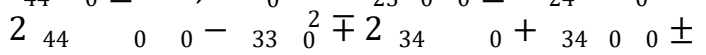

$$
\begin{aligned}
& 2440 \mp \text {, } \\
& \stackrel{\mp}{1}^{\mp}-23 \quad 0 \quad \pm 224 \quad 0-34^{2} \pm 2_{44} \\
& 33^{2} \pm 234 \quad-340 \pm 244 \quad 0 \mp, \quad 2= \\
& -230 \pm 224 \quad 0-34^{2} \pm 244 \quad 10 \frac{-}{\mp} \\
& 33^{2} \pm 234,-340, \pm 244,0 \mp, \quad \frac{\mp}{3}= \\
& \begin{array}{l}
, 230 \\
2{ }^{2} \mp 2,2 \\
33,
\end{array}
\end{aligned}
$$

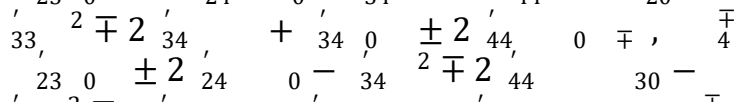

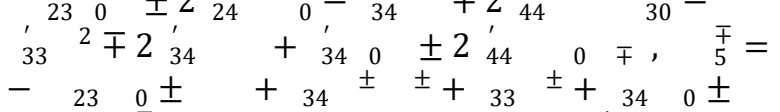

$$
\begin{aligned}
& , \quad \underset{6}{+}=-230 \pm \quad+34 \pm \frac{ \pm}{1}+33^{ \pm}+
\end{aligned}
$$

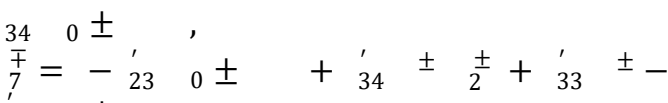

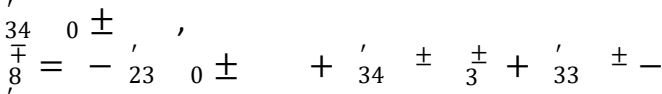

$$
\begin{aligned}
& 340 \pm \text {. }
\end{aligned}
$$

Solving Eq. (5.7), we get the ratios of the amplitude constants of irregular waves corresponding to the vertical components of the displacement.

$$
\frac{ \pm}{0}=\frac{ \pm}{ \pm}, \frac{ \pm}{0}=\frac{ \pm}{ \pm}, \frac{ \pm}{0}=\frac{ \pm}{ \pm}, \frac{ \pm}{0}=\frac{ \pm}{ \pm}
$$

where the values of $\quad \pm, \quad \pm, \pm$ and \pm are obtained by replacing first, second, third and fourth column of \pm with column matrix $\mathrm{T}^{\mp}$ respectively. The ratios of the amplitude constants of irregular waves corresponding to horizontal components of displacement are obtained with the help of Eqs. (3.5), (3.8) and (5.8) as

$$
\begin{gathered}
\frac{ \pm}{0}=\frac{ \pm}{0} \frac{ \pm}{ \pm}, \frac{ \pm}{0}=\frac{ \pm}{1} \frac{ \pm}{ \pm}, \frac{ \pm}{0}=\frac{\frac{ \pm}{2}}{0} \frac{ \pm}{ \pm}, \frac{ \pm}{0}= \\
\frac{{ }^{\frac{ \pm}{3}}}{0} \frac{ \pm}{ \pm} . \\
\text { (5.9) }
\end{gathered}
$$

The reflection and transmission coefficients for irregularly reflected and transmitted $\mathrm{qSV}$ and $\mathrm{qP}$-waves are

$$
\begin{aligned}
& \pm=\overline{\frac{1+ \pm^{2}}{1+{ }_{0}^{2}}} \frac{ \pm}{ \pm}, \\
& \pm=\overline{\frac{1+\frac{ \pm}{1}^{2}}{1+{ }_{0}^{2}}} \frac{ \pm}{ \pm} \\
& \pm=\frac{1+\frac{t^{2}}{1+\frac{2}{2}}}{ \pm} \frac{ \pm}{ \pm} \text {, } \\
& \pm=\frac{1+\frac{ \pm^{2}}{3}}{1+{ }_{0}^{2}} \frac{ \pm}{ \pm} \text {. }
\end{aligned}
$$

We come to know from Equation (5.10) that the coefficients corresponding to the irregularly reflected and 


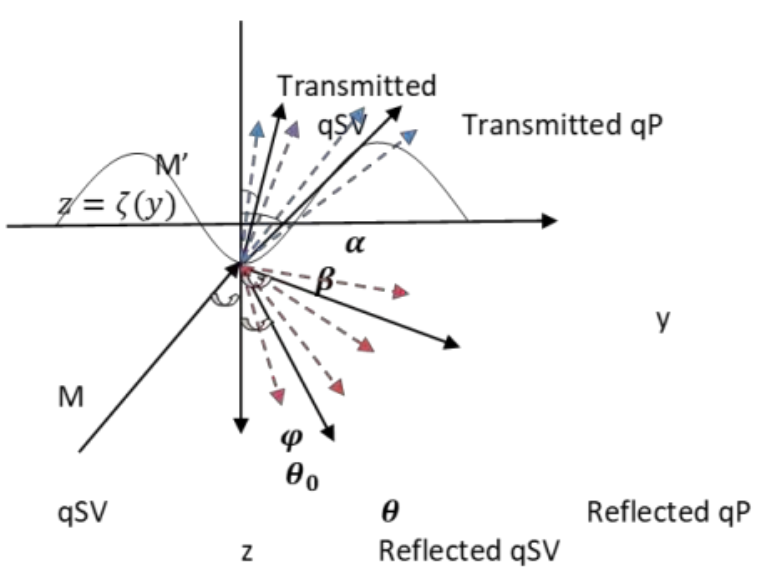

Figure 1: Geometry of the problem.

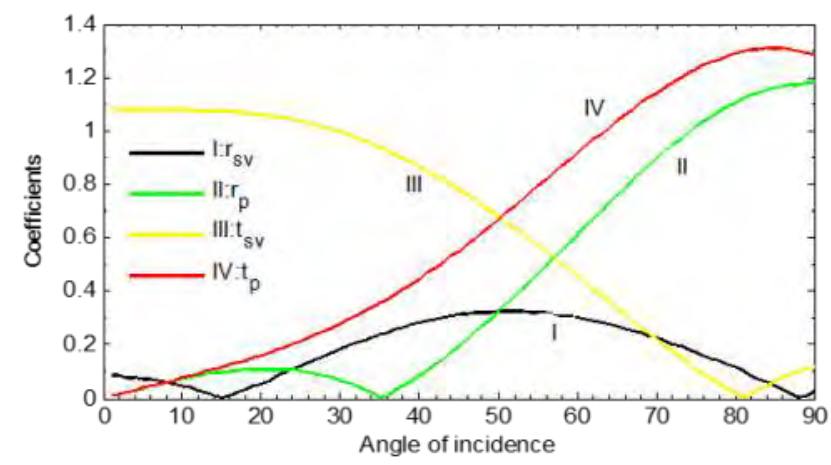

Figure 3: Variation of reflection and angle of incidence ( $\left.\theta_{0}\right)$.

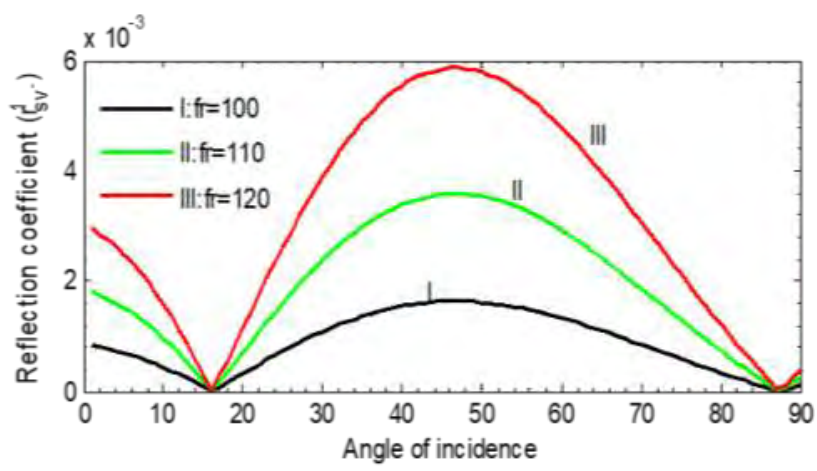

Figure 5: Variation of $r_{S V^{-}}^{1}$ with $\theta_{0}$ for different values of $\omega / p c_{0}$.

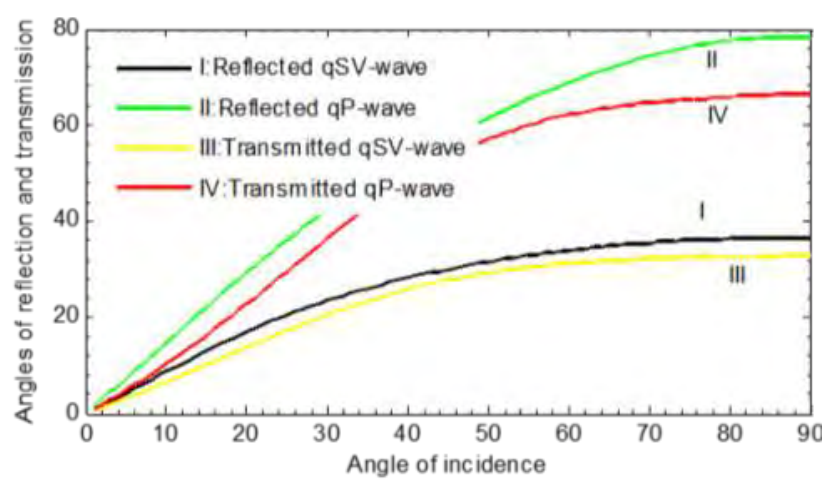

Figure 2: Variation of the angle of reflection and transmission coefficients with transmission with the angle of incidence.

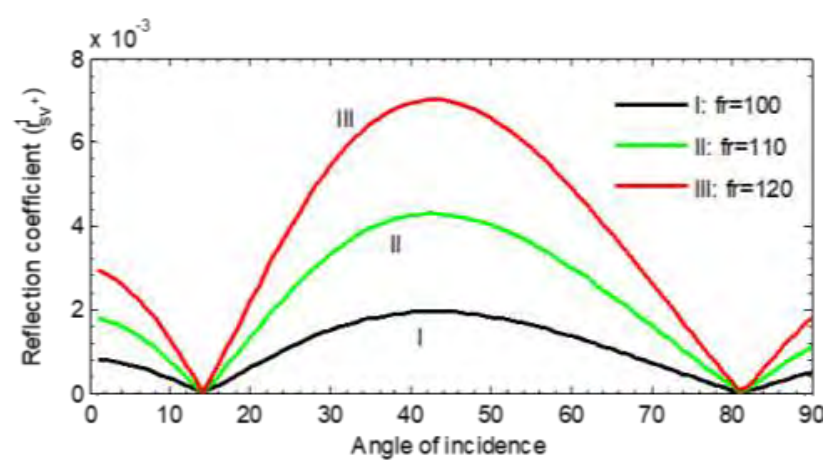

Figure 4: Variation of $r_{s V^{+}}^{\mathbb{1}^{+}}$with ${ }^{\theta_{0}}$ for different values of $\omega / p c_{0 x}$

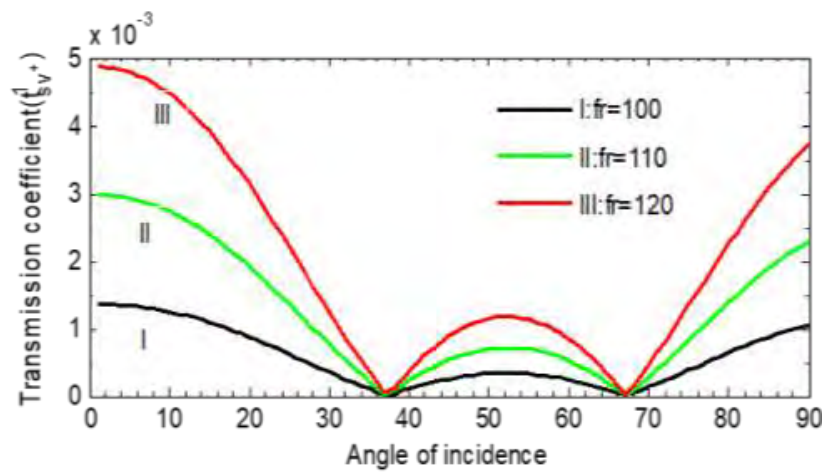

Figure 6: Variation of $t_{S V^{+}}^{1}$ with $\theta_{0}$ for different values of $\omega / p c_{0}$. 
We come to know from Equation (5.10) that the coefficients corresponding to the irregularly reflected and transmitted $q S V$ and $q P$-waves are functions of the elastic constants, angle of incidence, corrugation and frequency parameters.

\section{Special case:}

When the interface is represented by ${ }_{3}=\cos 2$ with $d$ as the amplitude of corrugation. In this case

$$
-==\begin{array}{ll}
0 & \neq 1 \\
\overline{2} & =1 .
\end{array}
$$

Thus, using these values, the reflection and transmission coefficients for the first order approximation of the corrugation are given by

$$
\begin{aligned}
& 1_{ \pm}=\overline{\frac{1+\frac{ \pm}{1}^{2}}{1+0_{0}^{2}}} \frac{\frac{ \pm}{1}}{ \pm}, \quad 1_{ \pm}= \\
& \overline{\frac{1+\frac{ \pm^{2}}{11}}{1+0_{0}^{2}}} \frac{\frac{ \pm}{1}}{ \pm} \\
& 1_{ \pm}=\overline{\frac{1+\frac{ \pm^{2}}{21}}{1+\frac{2}{2}}} \frac{\frac{ \pm}{1}}{ \pm}, \quad 1_{ \pm}= \\
& \overline{\frac{1+\frac{t^{2}}{31}}{1+{ }_{0}^{2}}} \frac{\frac{t}{1}}{ \pm}
\end{aligned}
$$

where values of $\frac{ \pm}{1}, \frac{ \pm}{11}, \frac{ \pm}{21}, \frac{ \pm}{31}, \pm \frac{ \pm}{1}, \frac{ \pm}{1}, \frac{ \pm}{1}$ and $\frac{ \pm}{1}$ are obtained from Eq.(5.10) by using Eq.(6.1). We will compute these coefficients for a particular model.

\section{Results and Discussion}

We will compute the angles of reflected and transmitted waves through Snell's law given by Eq. (3.9) in which the apparent velocity is related with dimensionless velocity by $=-$. Let us find out the angles of reflected qSV and qP-waves in the half-space, M. Eq. (2.8) may be written as

$$
{ }^{4}-\quad+2+\quad-2=0
$$

where

$$
\begin{aligned}
=\frac{}{244}, & =\frac{}{2 \frac{44}{2}}, \\
=\frac{44}{2}, & =\frac{}{44}
\end{aligned}
$$

There are two roots of ${ }^{2}$ corresponding to $\mathrm{qSV}$ and $\mathrm{qP}$ waves for a given $=\frac{3}{2}$, and for a given value of , there are two roots of $p$ corresponding to the angles of reflected $q S V$ and $q P$-waves [24]. Substituting the values of , and into Eq.(8.1), we get

$$
0^{4}+1^{3}+2^{2}+3+4=0
$$

where

$$
\begin{aligned}
& { }_{0}=33-{ }_{34}^{2}, \quad 1=2\left(\begin{array}{lll}
24 & 33 & -23 \\
34
\end{array}\right), \quad 2 \\
& =1+2233+22434-(1+23)^{2} \\
& -1+332 \text {, } \\
& \begin{aligned}
3=22234- & 2324-24+342,4 \\
& =4-1+22^{2}+22-24
\end{aligned}
\end{aligned}
$$

We transform this equation with $=\frac{1}{3}=\frac{2}{3}$ so that

$$
4^{4}+3^{3}+2^{2}+1+0=0
$$

This equation has two positive roots, i.e., the smaller positive root $\left({ }_{1}\right)$ and the larger positive root $(2)$ which represent the directions of reflected $\mathrm{qSV}$ and $\mathrm{qP}$-waves respectively. Thus, $=-1 \quad 1 \&=-1{ }^{-1}$ \& Similarly, in the half-space $\mathrm{M}^{\prime}$, the angles of transmitted $\mathrm{qSV}$ and $\mathrm{qP}$-waves are obtained as $=-1\left(\begin{array}{c}1 \\ 1\end{array}\right) \&=$

$$
-1\left(\begin{array}{c}
1 \\
2
\end{array}\right) \text {. }
$$

For the numerical computation, the following relevant values of elastic constants are taken Singh and Tomar (2007)

(for half-space, M-Lithium tantalate)

$$
\begin{aligned}
& { }_{24}=0.1110^{11}-2, \quad 23
\end{aligned}
$$

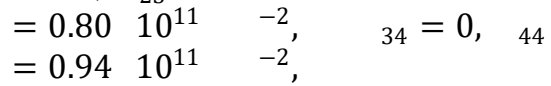

$$
\begin{aligned}
& \begin{array}{c}
33=2.7510^{11} \quad-2, \quad 22=2.33 \quad 10^{11}-2, \\
=7400 \quad-3 .
\end{array}
\end{aligned}
$$

(for half-space, $\mathrm{M}^{\prime}$-Lithium neobate) \।

$$
\begin{aligned}
& { }_{24}^{\prime}=-0.0910^{11} \quad-2,{ }_{23}^{\prime}=0.75 \quad 10^{11} \quad-2, \quad{ }^{\prime}
\end{aligned}
$$

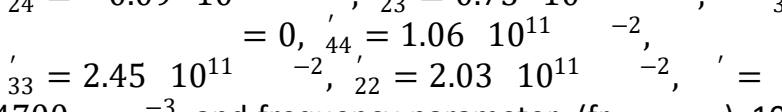
and corrugation parameter $(\mathrm{cor}=\mathrm{pd})=0.0001$ are taken unless and otherwise mentioned. Figure 2 represents the variation of angles of reflected and transmitted qSV and qP-waves with angle of incidence. Here all angles, $(,,$,$) of the regularly reflected and transmitted$ waves increase with the increase of angle of incidence $(0)$. It is observed that the angles of reflection and transmission for qSV-waves are less than that of the $\mathrm{qP}$ waves.

Curve I in Figure 3 shows that starts from certain value which decreases to zero at $0=15^{0}$ and then increases upto $0=51^{0}$ with the increase of 0 . Thereafter, it decreases, touching zero value at $0=87^{0}$. In the same figure, Curve II shows that is parabolic in the region $0 \leq$ $0 \leq 35^{0}$ and then, it increases with the increase of 0 . Curve III shows the decreasing nature of upto $0=$ $81^{0}$ and then increases with the increase of 0 , while Curve IV shows that increases with the increase of 0 .

\section{Effect of frequency parameters}

We are interested to see the effect of frequency parameters on the reflection and transmission coefficients. In Figure 4, the reflection coefficient, ${ }^{1}+$ corresponding to the irregularly reflected qSV-wave starts from certain value and decreases to zero at ${ }_{0}=14^{0}$ making a parabolic region in $14^{0} \leq 0 \leq 81^{0}$, which then increases with the increase of 0 . It is observed that ${ }^{1}+$ increases with the increase of frequency (fr) parameters. We have observed similar nature of ${ }^{1}$ - in Figure 5. 


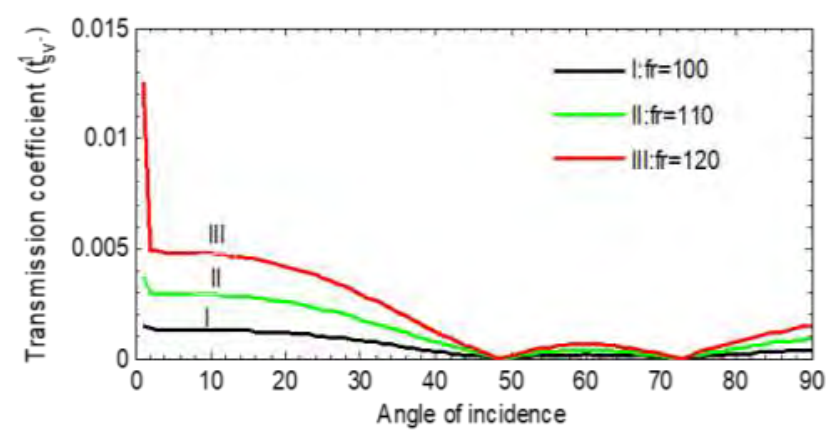

Figure 7: Variation of $t_{s V^{-}}^{1}$ with ${ }^{\theta_{0}}$ for different values of $\omega / p c_{0}$.

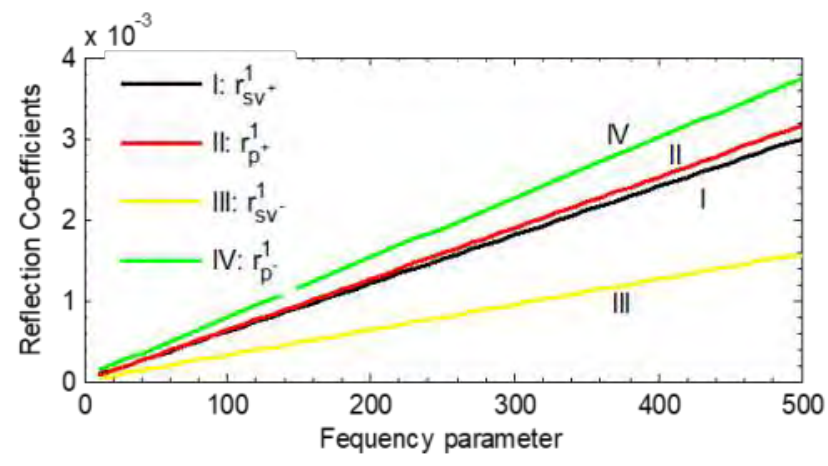

Figure 9: Variation of reflection coefficients of the irregularly reflected qSV \& $\mathrm{qP}$-waves with $\omega / p c_{0}$.

We are interested to see the effect of frequency parameters on the reflection and transmission coefficients. In Figure 4, the reflection coefficient, ${ }^{1}+$ corresponding to the irregularly reflected qSV-wave starts from certain value and decreases to zero at ${ }_{0}=14^{0}$ making a parabolic region in $14^{0} \leq{ }_{0} \leq 81^{\circ}$, which then increases with the increase of ${ }_{0}$. It is observed that ${ }^{1}+$ increases with the increase of frequency ( $\mathrm{fr}$ ) parameters. We have observed similar nature of ${ }^{1}$ - in Figure 5.

The coefficient, ${ }^{1}+$ in Figure 6 decreases initially making a parabolic region in $37^{0} \leq 0 \leq 67^{0}$ and then increases with the increase of 0 . It is observed that the values of ${ }^{1}+$ increase with the increase of frequency parameter. Similar natures of ${ }^{1}$ - are observed in Figure 7.

We have seen from Figure 8 that the coefficients corresponding to the regularly reflected and transmitted waves are independent of frequency parameters. In Figures 9 and 10, the coefficients corresponding to the irregularly reflected and transmitted waves are linearly proportional to the frequency parameters, but at different rates.

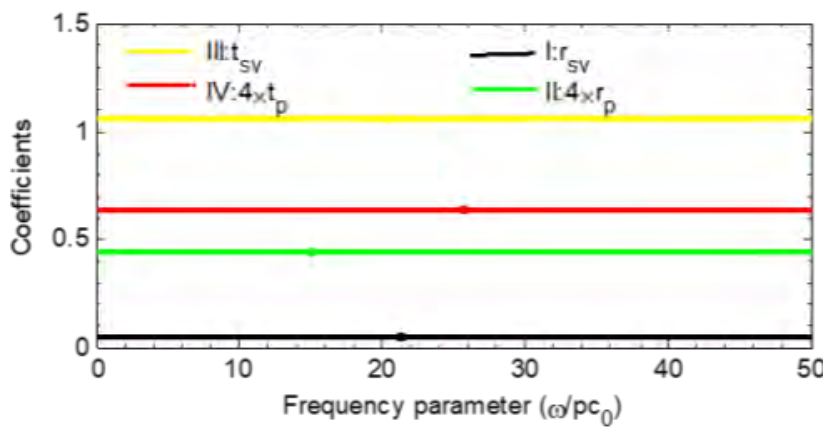

Figure 8: Variation of reflection and transmission coefficients of the regular qSV \& qP-waves with $\omega / p c_{0}$.

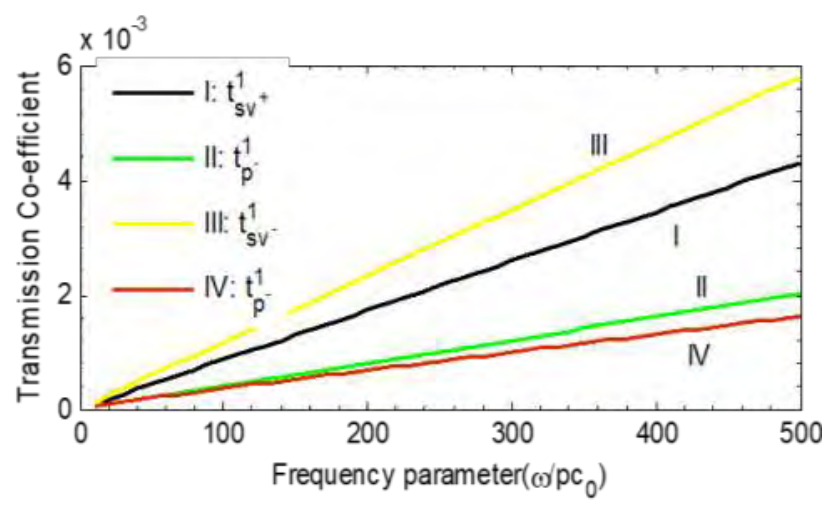

Figure 10: Variation of transmission coefficients of the irregularly transmitted qSV \& qP-waves with $\omega / p c_{0}$.

\section{Conclusion}

The problem of incident qSV-wave at a corrugated interface between two dissimilar monoclinic elastic halfspaces has been investigated. We have obtained the reflection and transmission coefficients for the first order of approximation corresponding to regularly and irregularly reflected and transmitted $\mathrm{qSV}$ and $\mathrm{qP}$-waves with the help of Rayleigh's method of approximation. These coefficients are computed numerically for a specific model and the effect of frequency parameters on these coefficients are discussed. We may conclude with the following remarks:

(i) All coefficients corresponding to regular waves are functions of angle of incidence and elastic constants, while those of irregular waves are found to be functions of angle of incidence, elastic constants and frequency parameters.

(ii) Theoretically and numerically, the reflection and transmission coefficients of the regular waves are independent of frequency parameters.

(iii) The coefficients corresponding to irregular waves 
are found to be linearly proportional to frequency parameters.

(iv) It is found that the values of coefficients corresponding to irregular waves increase with increase of frequency parameter.

(v) The values of coefficients corresponding to irregular waves are found to be small.

\section{REFERENCES}

Abubakar, I. (1962): Scattering of plane elastic waves at rough surfaces I. Proceedings of Cambridge Philosophical Society, 58, 136-157.

Asano, S. (1966): Reflection and refraction of elastic waves at a corrugated interface. Bulletin of Seismological Society of America, 56(1), 201-221.

Chattopadhyay, A., Choudhury S. (1995). The reflection phenomena of $\mathrm{P}$-waves in a medium of monoclinic type. International Journal of Engineering Science, 33 (2), 195-207.

Chattopadhyay, A., Saha, S., Chakraborty, M. (1997). Reflection and transmission of shear waves in monoclinic media. International Journal for Numerical and Analytic M ethods in Geomechanics, 21(7), 495-504.

Dowaik, M.A., Ogden, R.W. (1991). Interfacial waves and deformations in pre-stressed elastic media. Proceedings of the Royal Society of London, 433(A), 313-328.

Dunkin, J.W., Eringen, A.C. (1962). Reflection of elastic waves from the wavy boundary of a half space. Proceedings of the $4^{\text {th }}$ US National Congress of Applied M echanics. ASME Publication, pp. 143-160.

Gupta S.K. (1978). Reflections and refractions from curved interfaces: model study. Geophysical Prospecting, 26(1), 82-96.

Kuo, J.T., Nafe, J.E. (1962). Period equation for Rayleigh waves in a layer overlaying a half space with a sinusoidal interface. Bulletin of Seismological Society of America, 52(4), 807-822.

Kaur, J., Tomar, S.K. (2004). Reflection and refraction of SH-waves at a corrugated interface between two monoclinic elastic half-spaces. International Journal for Numerical and Analytic Methods in Geomechanics, 28(15), 1543-1575.

Levy, A., Deresiewicz, H. (1967). Reflections and transmission of elastic waves in a system of corrugated layers. Bulletin of Seismological Society of America, 57 (3), 393-419.
Rayleigh, L. (1907). On the dynamical theory of gratings. Proceedings of the Royal Society of London, 79(A), 399-416.

Rice, O. (1951). Reflection of electromagnetic waves from slightly rough surfaces. Communications on Pure and Applied M athematics, 4(2-3), 351-378.

Sheriff, R.E., Geldart, P.L. (1995). Exploration Seismology. Cambridge: Cambridge University Press.

Singh, S.J. (1999). Comments on "The reflection phenomena of $\mathrm{P}$-waves in a medium of monoclinic type by Chattopadhyay A and Choudhury". International Journal of Engineering Science, 37(3), 407-410.

Singh, S.J., Khurana, S. (2001). Reflection and transmission of P-and SV-waves at the interface between two monoclinic elastic half-spaces. Proceedings of the National Academy of Sciences, 71(A4), 305-319.

Singh, S.J., Khurana, S. (2002). Reflection of P and SVwaves at the free surface of a monoclinic elastic halfspace. Proceedings of Indian Academy of Sciences (Earth Planet Science), 111(4), 401-412.

Singh, S.S., Singh J. (2013). Effect of corrugation on incident qSV-waves in pre-stressed elastic half-space. International Journal of Applied Mathematics and Mechanics, 9(9), 92-106.

Singh, S.S., Tomar, S.K. (2007a). Quasi P-waves at a corrugated interface between two dissimilar monoclinic elastic half-spaces. International Journal of Solids Structure, 44(1), 197-228.

Singh, S.S., Tomar, S.K. (2007b). Elastic waves at a corrugated interface between two dissimilar fibrereinforced elastic half-spaces. International Journal for Numerical and Analytic M ethods in Geomechanics, 31(9), 1085-1116.

Singh, S.S., Tomar, S.K. (2008). qP-wave at a corrugated interface between two dissimilar pre-stressed elastic half-spaces. Journal of Sound and Vibration, 317(3-5), 687-708.

Tomar, S.K., Kaur, J. (2003). Reflection and transmission of $\mathrm{SH}$-waves at a corrugated interface between two laterally and vertically heterogeneous anisotropic elastic solid half-spaces. Earth, Planets Space, 55(9), 531-547.

Udias, A. (1999). Principles of Seismology. Cambridge: Cambridge University Press. 